\title{
A Fast-Marching Approach to Cardiac Electrophysiology Simulation for XMR Interventional Imaging
}

\author{
M. Sermesant ${ }^{1}$, Y. Coudière ${ }^{2}$, V. Moreau-Villéger ${ }^{3}$, K.S. Rhode ${ }^{1}$, \\ D.L.G. Hill ${ }^{4, \star}$, and R.S. Razavi ${ }^{1}$ \\ 1 Division of Imaging Sciences, King's College London, \\ $5^{\text {th }}$ floor Thomas Guy House, Guy's Hospital, London, UK \\ 2 Jean Leray Mathematics Laboratory, Nantes University, France \\ 3 Epidaure Research Project, INRIA, Sophia Antipolis, France \\ 4 Centre for Medical Image Computing, University College London, UK \\ derek.hill@ucl.ac.uk
}

\begin{abstract}
Cardiac ablation procedures are becoming more routine to treat arrhythmias. The development of electrophysiological models will allow investigation of treatment strategies. However, current models are computationally expensive and often too complex to be adjusted with current clinical data. In this paper, we have proposed a fast algorithm to solve Eikonal-based models on triangular meshes. These models can be used to extract hidden parameters of the cardiac function from clinical data in a very short time, thus could be used during interventions. We propose a first approach to estimate these parameters, and have tested it on synthetic and real data derived using XMR imaging. We demonstrated a qualitative matching between the estimated parameter and XMR data. This novel approach opens up possibilities to directly integrate modelling in the interventional room.
\end{abstract}

\section{Introduction}

The treatment of cardiac arrhythmias has changed considerably in the last fifteen years. Radio-frequency cardiac ablation techniques are becoming widely available as an alternative treatment to drug therapy. These are carried out under x-ray fluoroscopic guidance, with specialised catheters for making invasive recordings of the electrical activity in the heart, and even reconstruct the chamber geometry (CARTO from Biosense, EnSite from ESI).

These procedures can be highly effective with minimal side effects, but in some groups of patients have unsatisfactory success rates, are often very long, and can involve high x-ray radiation dose to both patient and staff. Moreover, serious side effects can arise if the lesions extend beyond the target area. There is a need for substantial innovation in order to reliably achieve successful results in an acceptable time, with lower radiation dose and reduced risk of accidental damage to adjacent structures.

^ Corresponding author.

J. Duncan and G. Gerig (Eds.): MICCAI 2005, LNCS 3750, pp. 607 615 2005.

(C) Springer-Verlag Berlin Heidelberg 2005 
The aim of this research work is to design models of the cardiac electrical activity that are suited for clinical use and to propose methods to combine these models with interventional data in order to better estimate the patient cardiac function and help in the guidance of procedures.

\section{$1.1 \quad$ Electrophysiology Models}

Modelling the electrophysiology of the cell is an active research area since the seminal work of Hodgkin and Huxley [1. The precise modelling of the myocardium involves a cell membrane model embedded into a set of partial differential equations (PDE) modelling a continuum. We can divide these models into three categories, from the more complex to the simpler (numerically):

- Biophysical models: semi-linear evolution PDE + ionic models. Up to fifty equations for ion concentrations and channels (Luo-Rudy, Noble)

- Phenomenological models: semi-linear evolution PDE + mathematical simplification of biophysical models. Reducing to two equations representing the intra- and extra-cellular potentials (bi-domain, mono-domain)

- Eikonal models: one static non-linear PDE for the depolarisation time derived from the previous models (Eikonal-Curvature, Eikonal-Diffusion)

Solutions of the evolution PDE are very computationally demanding, due to the space scale of the electrical propagation front being much smaller than the size of the ventricles. The motion of the front governed by the Eikonal equation is observed at a much larger scale, resulting in much faster computations.

For our interventional purpose, and as parameter adjustment often requires several simulations, we want to design a very fast model. Moreover, clinical data currently available is mainly on depolarisation times. For these reasons we chose to base the presented work on the Eikonal models. Even if these models are not able to precisely simulate the whole range of cardiac pathologies, they open up possibilities for fast estimation, filtering, interpolation and extrapolation. The long-term goal is to build up a hierarchy of models where a more complex model could be used in pathological areas.

\subsection{Clinical Measures}

XMR suites are a new type of clinical facility combining in the same room a MR scanner and a mobile cardiac x-ray set. The patient can be easily moved between the two systems using a specially modified sliding MR table top that docks with and transfers patients to a specially modified x-ray table. Techniques have been designed to register the two imaging spaces [2]. Therefore it is possible to combine patient anatomy with electrophysiology recordings.

\section{A Fast Electrophysiology Model}

The classical Eikonal equation is:

$$
c\|\nabla T\|=1
$$

where $T$ is the depolarisation time and $c$ is the local propagation speed. 
However, the propagation in an excitable medium like the myocardium depends on the curvature of the propagating front. It can be explained physically by the fact that if a convex front is propagating outward, the excitation of the neighbours is more spread out, so the excitation is slower than for a planar wave. Two different formulations have been proposed to introduce this effect. Both are based on asymptotic development of the solution around the activation front given by the Nagumo equation: $\partial_{t} u=D \Delta u+k f(u)$, where $u$ is the action potential. $f$ and $k$ describe the cell membrane, they refer to the ionic reactions in the cell. $D$ is the volumetric electrical conductivity of the tissue i.e. the transmission of the electrical wave from cell to cell. In the case of cardiac pathology, the conductivity and the ion channel mediated change of polarisation of the cardiac cells can be both involved.

The two resulting formulations are the Eikonal-Curvature equation [3]:

$$
c \sqrt{k D}\|\nabla T\|-D \kappa(T)=1
$$

with $\kappa(T)=\|\nabla T\| \operatorname{div}\left(\frac{\nabla T}{\|\nabla T\|}\right)$, and the Eikonal-Diffusion equation [4]:

$$
c \sqrt{k D}\|\nabla T\|-D \Delta T=1
$$

$c$ is a constant depending only on the function $f$.

\subsection{Fast-Marching Approach}

Different numerical approaches have been proposed to solve these equations. A temporal evolution term and finite differences have been applied to Eq. 2] [3]: $c \sqrt{k D}\|\nabla T\|-D \kappa(T)=\partial_{t} T$, and an evolution term and finite elements to the Eq. 3] [4]: $\partial_{t} T+c \sqrt{k D}\|\nabla T\|-D \Delta T=1$.

A time dependant PDE like these needs up to thousands of iterations, each of which might be a linear (or non-linear) system to solve. Furthermore, additional stability conditions constrain the computations. The static solving of Eikonal equations 2 and 3, first proposed by [5] with a Newton's method, requires to solve only one non-linear system of equations.

For sake of efficiency, our approach is also to directly solve the static equations 2 and 3, but takes advantage of the Fast-Marching Method (FMM) 6] to solve the non-linear system. FMM are numerical algorithms for solving Eq. 1 on a Cartesian grid in $O(M \log M)$ steps $\left(O\left(M^{2}\right)\right.$ for a Newton's method), where $M$ stands for the number of grid points. Consistent upwind discretisations of the gradient are used that select the correct viscosity solution, and leads to a causality relationship between the unknowns: the solution at a grid point depends only on the smaller adjacent points, and the unknown can be computed from point to point in an ascending manner.

We evaluate a first guess $T_{0}$ for $T$ by solving the Eq. 1 with the FMM, and then compute the curvature effect from $T_{0}$ to correct the equation and re-evaluate $T$. The iterative process is: $c\left\|T^{k+1}\right\|=1+F\left(T^{k}\right)$, where $F\left(T^{k}\right)=\kappa\left(T^{k}\right)$ for Eq. 2 and $F\left(T^{k}\right)=\Delta T^{k}$ for Eq. 3. The curvature term being only a small perturbation of the equation, the sequence $\left(T^{k}\right)$ hopefully converges quickly. Then its limit is a solution of the discretised equation. 


\subsection{Unstructured Grids Implementation}

The complexity of the heart geometry is difficult to describe with structured grids. Moreover, most of the 3D medical data is in the form of triangulated surfaces or point clouds. It is thus important to be able to deal with this kind of domain. This is why we implemented our algorithm on triangulations.

The FMM has been extended to unstructured grids, with criteria on the triangles to compute the narrow band values [7. We implemented Eq. 3] using using P1 Lagrange Finite Elements to compute the Laplacian. Experimental evidence suggests no flux on the myocardium surface, so we use Neumann boundary conditions. We integrate this in the stiffness matrix $K: K_{i j}=\int \nabla \phi_{i} \nabla \phi_{j}$ coming from an integration by parts of the Laplacian in the variational formulation, with $\phi_{i}$ and $\phi_{j}$ the P1 Lagrange shape functions.

We compute the curvature flow for Eq. 2 with the formula proposed in 8 . We adapted the edge-based formula into a triangle-based formula, faster for the neighbourhood iterators we are using, and to cope with the presence of holes in the mesh. The curvature flow at point $i$ is then:

$$
\kappa(T) \approx\|\nabla T\|_{i} \frac{\sum_{n \in \mathcal{N}_{i}} W_{j}^{i}\left(T_{j}-T_{i}\right)+W_{k}^{i}\left(T_{k}-T_{i}\right)}{\sum_{n \in \mathcal{N}_{i}} \operatorname{meas}(n)}
$$

with $\mathcal{N}_{i}$ the triangle neighbour set incident to $i ; j$ and $k$ the two other vertices of triangle $n$ (and $\alpha_{j}, \alpha_{k}$ the corresponding angles); $\|\nabla T\|_{i}$ the pointwise mass-lumped Galerkin approximation of the gradient in $i$; and $W_{j}^{i}=$ $\operatorname{cotan}\left(\alpha_{k}\right) /\left(2\|\nabla T\|_{n}\right)$; and meas $(n)$ the area of $n$.

Our $\mathrm{C}++$ implementation using the sorted containers of the Standard Template Library and precomputed neighbouring iterators makes it possible to compute the FMM and these terms in less than a second for a 13000 nodes mesh.

\subsection{Validation of the Algorithm: Convergence and Precision}

The numerical approach has been tested on Eq. 2 with $c=1$ and $\alpha=0.002$, on a family of unstructured meshes with up to 13092 nodes. Since we study the effect of curvature, the front is initially a circle of radius $r_{0}=0.1$ in the square $[-1,1] \times[-1,-1]$. As a consequence, the solution is expected to be mainly dependent on $r=\sqrt{x^{2}+y^{2}}$ so that its curvature $\kappa=1 / r$ varies from $1 / r_{0}=10$ down to 1 .

The computation of numerical solutions to Eq. 2 introduces errors when approximating the differential terms by differences on a mesh of nodes, and also when solving the resulting non-linear discrete problem.

We investigated numerically:

1. the algorithmic convergence of the sequence of approximation $T^{k}$ towards a limit, expected to be the solution $T_{N}$ of the discrete problem on a given mesh with $N$ nodes, Fig. 1 (Left);

2. the mesh convergence of the solution $T_{N}$ as $N \rightarrow \infty$, Fig. 1 (Right). 

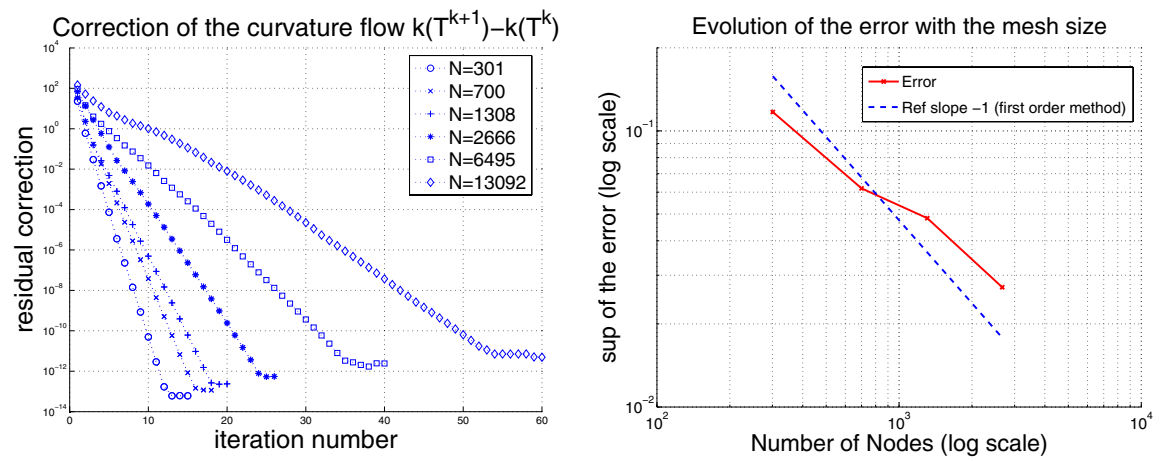

Fig. 1. (Left) Convergence of the algorithm for different mesh sizes. (Right) Evolution of the error with the mesh size.

Concerning point 1 , the sequence $T^{k}$ seems to converge, as shown in Fig. 11 Left. The number of iterations necessary for the residual correction $\left\|T^{k+1}-T^{k}\right\|_{\infty}$ to be of order $10^{-10}$ increase slowly as $N$, but remains reasonable: about 50 iterations on a 13092 nodes mesh, which means that 50 FMM solutions has been successively computed. Our FMM iterative algorithm looks like a good replacement for a Newton's method.

Concerning point 2, a reference solution is constructed on the finer mesh, and compared to the solutions on coarser meshes. As expected from the first order upwind differences used to discretise the gradient, the error decreases as $1 / N$ (ie as $h^{\frac{1}{2}}$ in 2D, standard for such methods). Of course higher order methods exist to discretise the gradient in an upwind manner. But, on unstructured meshes, they are not usually compatible with a Fast-Marching type algorithm.

\section{Local Apparent Conductivity Estimation}

Depolarization times on the endocardium are difficult to interpret due to the influence of the geometry and the curvature. The idea is to estimate hidden parameters using the proposed model and the clinical measurements in order to help diagnosis and therapy planning from electrophysiology study. A first step is to locate differences in local conductivity. In this section, we present a method to estimate this parameter using the fast Eikonal-Curvature model presented in the previous section (it could also be applied to the Eikonal-Diffusion).

Such parameters could eventually be estimated from the measured data using a signal processing approach. The model based method has the advantage of allowing the use of the model in a predictive way, once adjusted to the data. This can be very useful to test therapies and plan interventions. Moreover, it can be extended to partial observations: we aim to model the whole volumetric myocardium, but we will still have only access to surface data.

Estimating the parameters of a model from patient specific data is part of the field of data assimilation. Existing methods are generally based on the 
minimisation of the quadratic error between the model and the data: $\mathcal{C}(\mathbf{P})=$ $\sum_{i}\left(T_{i}^{m}-T_{i}^{s}(\mathbf{P})\right)^{2}$, where $T_{i}^{m}$ is the measured depolarisation time at vertex $i$ and $T_{i}^{s}(\mathbf{P})$ is the depolarisation time at vertex $i$ computed with the set of parameters $\mathbf{P}$. The minimisation is either sequential like in Kalman filtering based methods or global like in adjoint methods.

The time introduced in Eq. 3 or 2 to solve the Eikonal equation is an artificial time, therefore the sequential methods are not the natural framework to solve this problem. Moreover sequential methods involve the updating of all the variables and of a covariance matrix of the same size, involving additional computational costs. The adjoint methods propose a precise method to compute the gradient of $\mathcal{C}$ but the numerical scheme proposed, alternating a fast marching step and the computation of diffusion/curvature term, implies that the adjoint methods are not directly applicable to this problem.

The adaptation of one of these methods to the proposed discrete model is still work in progress. As a first approach, we proposed to adjust the conductivity parameter $D$ by iterating the two following steps:

- convergence of the Eq. 2 using presented algorithm

- local adjustment by multiplying $D$ by $1+\varepsilon$ or $1-\varepsilon$ depending on the sign of the difference between the measured and simulated depolarisation times

We expect this parameter to be different (smaller) in pathological regions.

\subsection{Synthetic Data}

We simulated on a triangulated sphere different propagation conditions to test the estimation procedure. On this normalised test, $c_{0}=1, k=1, D_{0}=0.01$. We defined three different zones: one excitation zone for the initialisation, one zone where $D=2 D_{0}$, and one zone where $D=D_{0} / 2$. We simulated the propagation and stored the depolarisation times. We then started the data assimilation procedure with $D=D_{0}$.

After convergence of this procedure, we obtain a mean error of $2.95 \times 10^{-3}$ and a maximum error of $8.46 \times 10^{-3}$ on the depolarisation times which are between 0 and 31 (arbitrary units). We were thus able to detect very precisely the areas with different conductivity and these conductivities were well estimated (cf Fig. 2).

\subsection{XMR Interventional Data}

XMR registration makes it possible to integrate in a same coordinate space the electrophysiology measurements and the patient anatomy. This opens up possibilities to obtain very rich data to validate the estimation procedure, as MR can give spatial information on the location of the pathology.

We used measurements from the Ensite system (Endocardial Solutions), which is a non-contact invasive catheter based device for recording the electrical activity of the heart (reconstructed on 256 points). Due to the tangential aspect of the fibre orientations, we believe that the 3D aspect of the propagation 

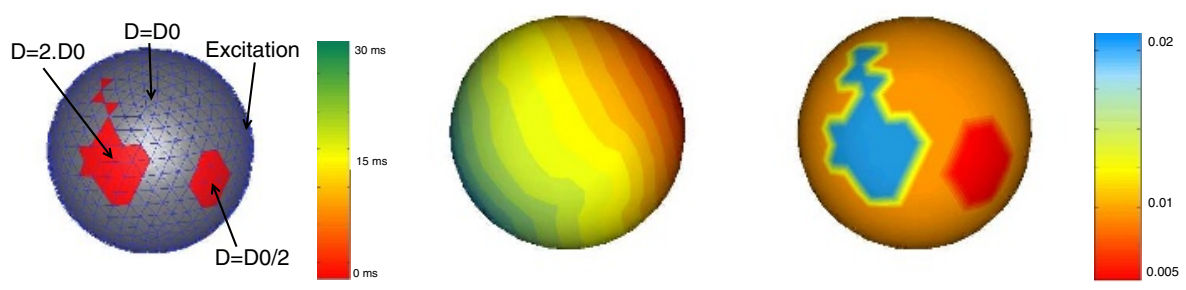

Fig. 2. (Left) Simulation of different conduction zones with $D$ twice the normal value (left zone) and half the normal value (right hexagonal zone). (Middle) Resulting isochrones with the Eikonal-Curvature equation. The excitation zone is on the right side of the sphere. (Right) Estimated $D$ with the parameter estimation procedure.
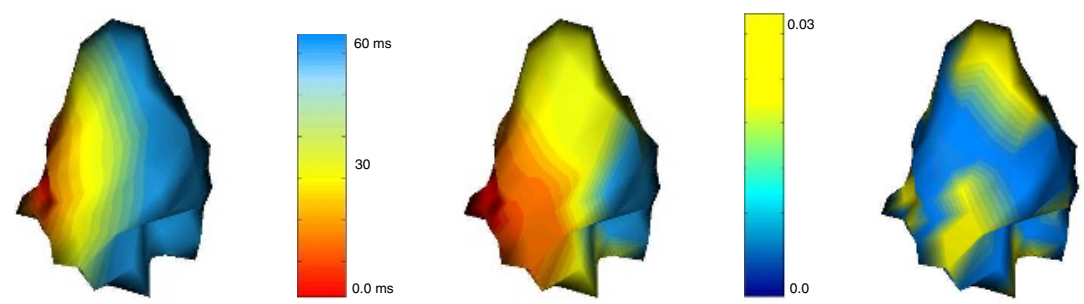

Fig. 3. (Left) Initial propagation with standard parameters. (Middle) Resulting isochrones with adjusted the Eikonal-Curvature equation . (Right) Estimated $D$ with the parameter estimation procedure. Mesh: 256 nodes.
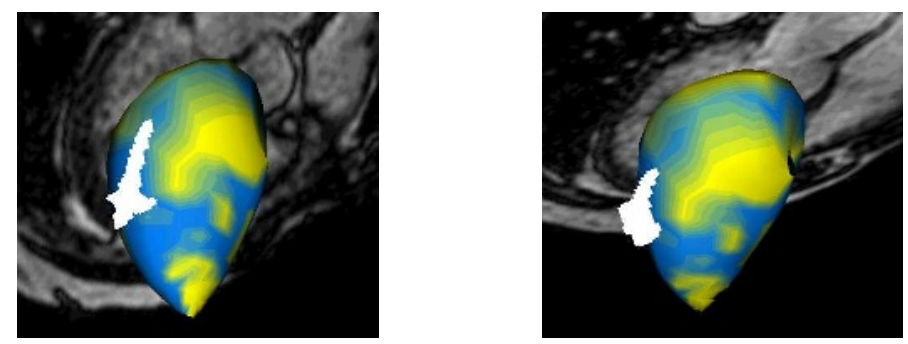

Fig. 4. Matching between the conductivity estimated with the described procedure and a scar segmented in a late enhancement MR image (white voxels). The complete $\mathrm{XMR}$ registration involves non-rigid deformation, this is why the shape of the basket is different between Fig. 3 and Fig. 4

does not interfere too much with the surface endocardial recordings. This data is from a patient with a left bundle branch block, so the initialisation does not come from the Purkinje network, but through the septum. From these recordings, we initialise the depolarisation in the model. We then adjusted the local conductivity. We obtain a mean error of $7.75 \times 10^{-1} \mathrm{~ms}$ and a maximum error of $9.75 \mathrm{~ms}$ on the depolarisation times which are between 0 and $66 \mathrm{~ms}$. 
The XMR registration makes it possible to locate these electrophysiology measurements in the MR coordinate space. This patient had multiple scars in the myocardium, leading to a left bundle branch block and poor cardiac function. Using late enhancement MR, some of these scars were manually segmented by an expert. The local apparent conductivity estimation procedure can then be compared with the scars locations. Precise comparison is still work in progress, but the first results obtained compare qualitatively well with the segmented scar. We can see in Fig. 4 that the segmented scar corresponds to a lower apparent conductivity zone (blue).

The mean value of the adjusted local apparent conductivity is 0.0149 and the maximum value is 0.0309 .

\section{Conclusion}

We presented in this article a new algorithm to achieve fast simulations of electrophysiology, along with a procedure to adjust the model parameters from interventional data. This algorithm has been validated on analytical solutions and the procedure has been tested on synthetic and real data. It was used to estimate the local apparent conductivity from interventional data, and the first results obtained are very encouraging. Having such a model opens up possibilities for real-time filtering and interpolation of electrophysiology recordings. Moreover, hidden parameter estimation is of great use for a better evaluation of the extent of the pathology and for planning of the therapy . An excellent example application is the planning of bi-ventricular pacing therapy for treatment of heart failure. A model with accurate information on the local conductivity would allow a better placement of the pacing leads and thus man improve of the 50-60\% success rate of the procedure.

This simulation part could be improved by using higher order schemes for the FMM and the curvature term, and the improvement of the implementation in case of obtuse angles in the triangulation. The current implementation does not treat these cases separately, but the numerical schemes should be different. Also, including the fibre orientations, which is rather straightforward in this formulation, could give more reliable results. We also plan to study more sophisticated estimation procedures, to achieve a precise and robust adjustment. In particular, we want to study the possibility to adapt a sequential method, like unscented Kalman filtering, or adjoint methods to this parameter estimation problem.

\section{References}

1. Hodgkin, A., Huxley, A.: A quantitative description of membrane current and its application to conduction and excitation in nerve. J. of Physio. 177 (1952) 500-544

2. Rhode, K., Hill, D., Edwards, P., Hipwell, J., Rueckert, D., Sanchez-Ortiz, G., Hegde, S., Rahunathan, V., Razavi, R.: Registration and tracking to integrate X-ray and MR images in an XMR facility. IEEE Trans. on Med. Imaging 22 (2003)

3. Keener, J., Sneyd, J.: Mathematical Physiology. Springer (1998) 
4. Franzone, P.C., Guerri, L., Rovida, S.: Wavefront propagation in activation model of the anisotropic cardiac tissue. J. Math. Biol. (1990)

5. Tomlinson, K., Hunter, P., Pullan, A.: A FEM for an eikonal equation model of myocardial excitation wavefront propagation. SIAM J. Appl. Math. (2002)

6. Sethian, J.: Level Set Methods and Fast Marching Methods. CUP (1999)

7. Kimmel, R., Sethian, J.: Computing geodesic paths on manifolds. Proceedings of National Academy of Sciences 95 (1998) 8431-8435

8. Barth, T., Sethian, J.: Numerical schemes for the Hamilton-Jacobi and Level Set equations on triangulated domains. J. of Computational Physics 145 (1998) 1-40 